\title{
Hypo-collagenesis in photoaged skin predicts response to anti-aging cosmeceuticals
}

\author{
Dana L Sachs, MD, Laure Rittié, PhD, Heather A Chubb, MS, Jeffrey Orringer, MD, \\ Gary Fisher, PhD, \& John J Voorhees, MD
}

Department of Dermatology, University of Michigan, Ann Arbor, Michigan, USA

Summary

Correspondence: D L Sachs, 1910 Taubman Center, 1500 E. Medical Center Drive, SPC 5314, Ann Arbor, MI 48109-5314, USA. E-mail: dsachs@med.umich.edu

This paper was presented at the $72^{\text {nd }}$ Meeting of the Society for Investigative Dermatology, Raleigh, North Carolina, May 2012.

This paper was funded in full by the University of Michigan Department of Dermatology.

Accepted for publication January 24, 2013

\begin{abstract}
Background Chronic sun exposure causes photoaging, the appearance of prematurely aged skin. This phenomenon is characterized by progressive alteration of the dermal extracellular matrix, including elastin and collagen fibers. While many cosmeceuticals claim to improve the appearance of photoaged skin, data are lacking regarding their ability to induce molecular responses associated with wrinkle effacement, particularly increased collagen production.

Aims To conduct a meta-analysis to determine whether there was a factor(s) that could predict response to various cosmeceuticals.

Patients/Methods Hundred subjects enrolled in five separate studies of cosmeceuticals containing: L-ascorbic acid, pentapeptide, $\alpha$-lipoic acid, yeast extract, or $1 \%$ idebenone. Five groups consisting of 16-20 volunteers applied one cosmeceutical to their photodamaged forearms for several weeks. Punch biopsies were obtained pretreatment and post-treatment and analyzed for type I procollagen by ELISA.

Results Analysis of basal collagenesis reinforced the notion that hypo-collagenesis is associated with photoaging severity, independent of age or gender. Treatment outcome varied greatly among subjects, ranging from no improvement to a 7 -fold increase in collagenesis. Retrospective statistical meta-analysis was conducted to determine whether age, gender, type of cosmeceutical, or evidence of hypocollagenesis in untreated skin could predict responsiveness to cosmeceuticals. Our analysis revealed that subjects with hypo-collagenesis responded 6.4 times more often than subjects with normo-collagenesis.

Discussion Hypo-collagenesis was the only factor that influenced treatment outcome. This study therefore identifies hypo-collagenesis as the unique parameter predicting anti-aging cosmeceutical treatment outcome. These findings provide a basis for future cosmetic testing and the potential development of custom formula skin care.
\end{abstract}

Keywords: aging, photoaging, cosmeceutical, collagen, vitamin C, photodamage

\section{Introduction}

Photoaging is characterized by dramatic alterations in the appearance of skin, for example presence of fine and deep wrinkles (rhytides), sallowness, mottled pigmentation, age or brown spots (lentigines), and broken blood vessels (telangiectasia). Biochemically, photoaged skin is characterized by the presence of damaged 
extracellular matrix components including elastin and type I collagen fibers, the latter being the most abundant protein in the dermis. ${ }^{1,2}$ Actinic collagenolysis, break-down of type I collagen fibers due to chronic sun exposure, is initiated by ultraviolet (UV) irradiationinduced matrix metalloproteinase activity that fragments dermal collagen fibers. ${ }^{3}$ Alongside matrix metalloproteinase activation, UV irradiation prevents dermal repair by transiently inhibiting procollagen production by resident fibroblasts. ${ }^{4}$ Collagen fragmentation accumulates in chronically sun-exposed skin due to repeated UV irradiation exposure. As actinic collagenolysis progresses to advanced stages, the mechanical tension in the dermis decreases. Dermal fibroblasts respond to the decreased mechanical tension by reducing their ongoing collagen production. ${ }^{5}$ In severely photoaged skin, collagen fragmentation is widespread, mechanical tension is largely reduced in the dermis, and therefore, fibroblasts are in a self-sustained hypocollagenesis state. ${ }^{6}$ Based on this degenerative process, an important and logical strategy for skin rejuvenation is to stimulate collagen production in photoaged skin.

Albert Kligman first described cosmeceuticals as products that may improve the appearance of photoaged skin, without the need to abide by the regulatory standards of a drug. ${ }^{7}$ Lying somewhere between a cosmetic and a pharmaceutical, these preparations have garnered tremendous interest for their potential to improve the appearance of photoaged skin without the need for a medical prescription. Adding to consumer appeal, cosmeceuticals are generally less expensive than prescription drugs for photoaged skin. Cosmetic procedures such as dermal fillers, botulinum toxin injections, and ablative laser resurfacing are more expensive and come with a longer recovery period than topical therapy. Although topical retinoids have been the mainstay of topical therapy for photoaged skin, their use is associated with varying degrees of erythema and irritation. ${ }^{8}$ Thus, there is great interest in developing agents that could mimic the wrinkle effacement of topical retinoids without the associated side effects. As a result, a plethora of topical cosmeceuticals are continually being developed. Yet, extremely few studies have independently validated the efficacy of these agents to improve the effects of photoaging in human skin in vivo.

Given that we and others have shown that improvement of the appearance of photoaged skin strongly correlates with increased collagenesis, as measured by ongoing synthesis of the type I collagen precursor procollagen $\mathrm{I},{ }^{6,8,9}$ we set out to test the ability of several, commercially available cosmeceuticals to increase collagenesis in photoaged skin in vivo. Interestingly, we found that all five tested cosmeceuticals were effective only in a subset of treated individuals. Statistical meta-analysis of nearly one hundred treated subjects was conducted to identify factors distinguishing between responders and non-responders among age, sex, cosmeceutical, and hypo-collagenesis condition. Altogether, our results demonstrate that hypocollagenesis is a strong indicator for positive response of photoaged skin to anti-aging cosmeceuticals.

\section{Material and methods}

\section{Material and treatment regimens}

Five cosmeceuticals were purchased and used with the regimen recommended by their respective manufacturer as follows: vitamin $\mathrm{C}$ cream (Active $\mathrm{C}^{\circledR}$, La Roche-Posay, L'Oréal, Paris, France), $\alpha$-lipoic acid cream (Antioxidant \& Texturizing Day Cream with Alpha lipoic acid, Reviva labs, Haddonfield, NJ, USA), and live yeast extract cream (Preparation $\mathrm{H}$ with Bio-Dyne, WhiteHall Robbins-Pfizer Canada, Kirkland, Quebec, Canada) were applied twice daily for 2 weeks; collagen-derived pentapeptide cream (StriVectin SD ${ }^{\circledR}$, Strivectin Operating Company, New York, NY, USA) was applied three times a day for 2 weeks; and $1 \%$ idebenone (anti-oxidant) cream (Prevage ${ }^{\circledR} \mathrm{MD}$, Allergan, Irvine, CA, USA) was applied twice daily for 10 days.

\section{Subject description and treatment}

Each study was approved by the Institutional Review Board of the University of Michigan according to the Declaration of Helsinki protocols, and written informed consent was obtained from each subject before enrollment. In all, 100 human subjects aged over 45 years were enrolled, irrespective of ethnicity or gender. None had an occupation (or other reason) that prevented typical chronic sunlight exposure. All subjects showed clinical features of photodamage on their forearms (such as leathery appearance or mottled pigmentation), albeit to various extents. Four subjects did not complete their respective studies due to various degrees of irritation (details in Table 1), and five subjects were eliminated due to substandard tissue processing in the laboratory. In the end, 91 subjects were analyzed for procollagen I (33 men and 58 women, age 49-82 years, mean age 65.3).

Topical treatments were performed on the extensor surface of one forearm selected via computer-generated randomization. Each subject was instructed to gently 
Hypo-collagenesis predicts response to cosmeceuticals - D L Sachs et al.

Table 1 Human subject distribution

\begin{tabular}{llll}
\hline Active Ingredient $^{\dagger}$ & No of subjects & \% of total & Dropped* $^{*}$ \\
\hline Vitamin C & 19 & 21 & $1^{4}$ \\
-lipoic acid & 16 & 17.5 & 0 \\
Live yeast extract & 20 & 22 & $1^{5}$ \\
$1 \%$ idebenone & 16 & 17.5 & $2^{2 \&(3+4)}$ \\
Pentapeptide & 20 & 22 & 0
\end{tabular}

*Number of research subjects that were enrolled but did not complete the study; not taken into account in final subject count.

${ }^{\dagger}$ As described by manufacturer.

Reasons for treatment discontinuation were ${ }^{2}$ rash, ${ }^{3}$ appearance of bumps, ${ }^{4}$ itching at site of treatment, or ${ }^{5}$ tingling in throat.

wash his or her selected forearm with soap and water, rinse with clear water, thoroughly dry the area, apply $\sim 750 \mu \mathrm{L}$ of the cosmeceutical preparation (measured with 5 "pumps" for idebenone or with a level spoon for all other cosmeceuticals), and rub gently until cream was absorbed. The first application was supervised by a nurse. Subjects kept daily journals of their applications during the remainder of the study, and containers were weighed before and after the study to verify compliance.

Treatments were generally well tolerated. Six subjects reported irritation without withdrawing from the study. Among these six subjects, five had received 1\% idebenone cream $(5 / 16=31.3 \%$ of idebenone creamtreated subjects) and three of which (60\% of affected subjects, $18.8 \%$ of idebenone subjects) were treated with topical steroid $(0.1 \%$ triamcinolone) after the trial period, until irritation resolved (within 1 week). The sixth subject had received collagen-derived pentapeptide cream $(1 / 20=5 \%$ of pentapeptide cream-treated subjects) and also received topical steroid treatment until normalization.

\section{Biopsy procurement}

For each treated subject, one 4-mm punch biopsy was taken at baseline before treatment, and another biopsy was taken $24 \mathrm{~h}$ after the last treatment. Skin samples were immediately embedded in Tissue-Tek OCT compound (Miles, Naperville, IL, USA), frozen in liquid nitrogen, and stored at $-80^{\circ} \mathrm{C}$ until processing.

Protein extraction and procollagen I enzyme-linked immunosorbent assay (ELISA)

Soluble proteins were extracted from frozen skin samples and analyzed for type I procollagen by ELISA as previously described. ${ }^{10,11}$ Procollagen I protein concentrations were normalized to dermal volume ${ }^{10,11}$ and presented as $\mathrm{ng} / \mathrm{mm}^{3}$. Fold change describes the ratio after/before treatment.

\section{Statistical analysis}

Statistical analyses were performed using the SAS-9.2 software (SAS Institute, Inc., Cary, NC, USA). Cochran-Mantel-Haenszel $(\mathrm{CMH})$ tests were used to determine statistically significant differences between subpopulations, controlling for treatment in the combined-study meta-analysis. Corresponding odds ratios were reported. Additionally, a sensitivity analysis was performed using the $\mathrm{CMH}$ test to investigate whether the meta-analysis had introduced a bias. Differences in treatment outcome measurements were assess using the Wilcoxon's signed-rank test within similar treatments. Pearson's correlation coefficients were used to describe the relationship among age, collagenesis levels, and/or procollagen fold change. An overall alpha-level of 0.05 was used to determine statistical significance, and all statistical tests were two-sided. Summary data are presented in bar graphs as means \pm standard error of the mean (SEM).

\section{Results and discussion}

\section{Description of the setting}

The main aim of this work was to test the ability of several anti-aging cosmeceuticals to increase collagen synthesis in photoaged skin in vivo. For each subject, a punch biopsy was obtained from the forearm at baseline and after treatment to measure procollagen I levels and determine response to treatment. Five cosmeceuticals were tested independently. Results were similar for each of the cosmeceuticals: we observed a large interindividual variation in response, with a mix of subjects whose procollagen I levels increased upon treatment (responders) and subjects with no increase (nonresponders) within each study. As shown in the metaanalysis, none of the cosmeceuticals were driving the results. To better understand intersubject variability, we conducted detailed statistical analysis to determine whether any criterion was associated with the responsiveness that was observed in a subset of treated subjects.

\section{Study 1: L-ascorbic acid}

The study of vitamin C (L-ascorbic acid) is illustrative of our findings with the other four cosmeceuticals. 
Topically applied vitamin $\mathrm{C}$ has been shown to increase procollagen I production ${ }^{12}$ and improve the clinical appearance of photoaged skin. ${ }^{13}$ When attempting to reproduce these findings with nineteen subjects, we found modest and no significant averaged changes in procollagen I levels upon treatment (1.46 \pm 0.25 -fold change vs. baseline, $n=19$, $P=0.515)$ Figure 1a. In fact, a subset of the treated subjects did not show any change at all, consistent with what was reported by others. ${ }^{12}$ Closer analysis of the data revealed a relatively large interindividual variation in baseline collagenesis as measured by procollagen I protein levels, which ranged from 1.8 to $43.6 \mathrm{ng} / \mathrm{mm}^{3}($ mean $=17.2$, median $=16.4, n=19)$. The subjects with hypo-collagenesis (defined as baseline procollagen I levels below median value, $n=9$ ) displayed significant and relatively robust increases in skin procollagen I protein levels upon treatment (2.01 \pm 0.44 -fold increase $n=9, P=0.039)$. In contrast, the subset of subjects with baseline levels above median value did not display any increase in procollagen I levels relative to baseline $(0.97 \pm 0.19$-fold vs. baseline, $n=10, P=0.193$; Fig. 1b). Furthermore, the amount of increased procollagen I after treatment significantly correlated with the subjects' basal collagenesis levels (Pearson's correlation coefficient $=-0.45$, $n=19, P=0.05$; Fig. 1c). Taken together, our data demonstrate that topical vitamin $\mathrm{C}$ increases procollagen I production in the skin of a subset of individuals, and that the treatment outcome is positive in subjects with hypo-collagenesis, defined as "below average" procollagen I synthesis levels in untreated skin.

\section{Meta-analysis of five cosmeceuticals}

The outcome of the vitamin C study was illustrative of our general results with four other anti-aging cosmeceuticals. However, to increase the strength of our findings, we retrospectively analyzed results from other University of Michigan studies with similar objectives and design. Each study was conducted independently, and human subjects were enrolled sequentially in each study. Lack of subject randomization among studies prevented direct statistical comparison of treatment efficacy (although each treatment outcome was similar to the one presented in Fig. 1a for topical vitamin C). Other cosmeceuticals tested respectively contained as their main active ingredient: $\alpha$-lipoic acid, idebenone, live yeast extract, or a collagen-derived pentapeptide. Detailed characteristics of the cohorts are summarized in Table 2. In all, results from 91 subjects were submitted to retrospective meta-analysis in order to deter-
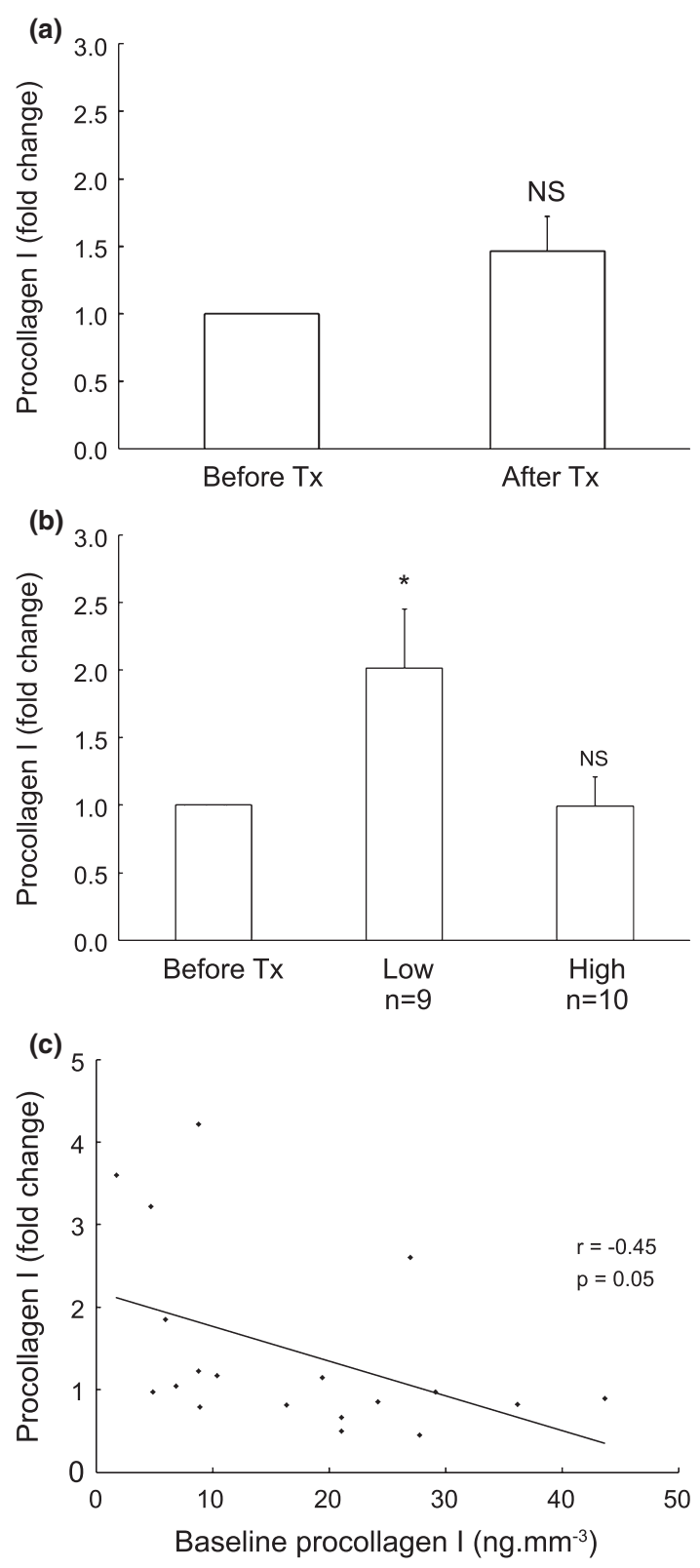

Figure 1 The procollagen I-enhancing effect of topical vitamin C in photoaged skin is evident in subjects with hypo-collagenesis (a) Skin procollagen I levels before and after topical vitamin C treatment (Tx) as measured by ELISA $(n=19)$ (b) Results from (a) were split in groups based on subjects' basal procollagen I synthesis levels as described in text. NS, nonsignificant; *: $P<0.05$ vs. Before Tx (c) Pearson's test shows correlation between basal collagenesis levels and improvement (correlation coefficient $=-0.45, n=19, P=0.05$ ).

mine whether differences existed between outcomes (increased procollagen I levels) and type of cosmeceutical, age, gender, or evidence of hypo-collagenesis in untreated photoaged skin. Changes in procollagen I 
Table 2 Human subject characteristics

\begin{tabular}{|c|c|}
\hline Variable & Description \\
\hline Gender & $\begin{array}{l}\text { Males: } 33(36 \%)^{*} \\
\text { Female: } 58(64 \%)^{*}\end{array}$ \\
\hline Treatment & $\begin{array}{l}\text { Vitamin C cream: } 19(21 \%)^{*} \\
\alpha \text {-lipoic acid cream: } 16(17.5 \%)^{*} \\
\text { Live yeast extract cream: } 20(22 \%)^{*} \\
1 \% \text { idebenone cream : } 16(17.5 \%)^{*} \\
\text { Collagen-pentapeptide cream: } 20(22 \%)^{*}\end{array}$ \\
\hline Age (in years) & $65.3(0.95)[49-82]^{\dagger}$ \\
\hline $\begin{array}{l}\text { Procollagen levels } \\
\text { at baseline }\left(\mathrm{ng} / \mathrm{mm}^{3}\right)\end{array}$ & $14.5(0.95)[1.8-43.7]^{\dagger}$ \\
\hline $\begin{array}{l}\text { Low baseline } \\
\qquad\left(\leq 15 \mathrm{ng} / \mathrm{mm}^{3}\right)\end{array}$ & $53(58 \%)^{*}$ \\
\hline $\begin{array}{l}\text { High baseline } \\
\left(>15 \mathrm{ng} / \mathrm{mm}^{3}\right)\end{array}$ & $38(42 \%)^{*}$ \\
\hline
\end{tabular}

*Number of subjects (\% of total).

${ }^{\dagger}$ Mean (SE) [range].

levels were expressed as increased (defined as fold change vs. baseline $>1.2$ ) or not increased (fold change vs. baseline $\leq 1.2$ ). The 1.2 threshold was defined to allow for $20 \%$ variation in accuracy for tissue processing and procollagen measurement.

As observed in the vitamin $\mathrm{C}$ cohort, collagenesis levels at baseline were variable among subjects. The mean procollagen I levels at baseline for all studies ranged from 1.8 to $43.7 \mathrm{ng} / \mathrm{mm}^{3}$ and averaged $14.5 \mathrm{ng} / \mathrm{mm}^{3}$. We defined the $95 \%$ confidence interval of the mean $\left(12.6-16.4 \mathrm{ng} / \mathrm{mm}^{3}\right)$ as intermediate procollagen I levels. Nineteen subjects $(21 \%)$ had intermediate collagenesis levels; 40 subjects (44\%) had hypo-collagenesis (defined as baseline procollagen I levels $<12.6 \mathrm{ng} / \mathrm{mm}^{3}$ ), and 32 subjects (35\%) were characterized by normo-collagenesis (defined as baseline procollagen I levels $>16.4 \mathrm{ng} / \mathrm{mm}^{3}$ ). We found that hypo-collagenesis was not correlated with age or gender (Fig. 2). These results further reinforce the notion that hypo-collagenesis in photoaged skin reflects the intensity of chronic sun exposure ${ }^{9}$ and thus varies among individuals based on their sun exposure history (and possibly other factors), not on their age or gender.

Statistical analysis was methodically conducted to determine which, if any, of the four criteria tested (type of cosmeceutical, age, gender, or hypo-collagenesis) were statistically associated with treatment outcome (i.e., increased procollagen I levels). Results are presented in Table 3. In all, we found no relationship between treatment outcome and age (Pearson's correlation, $P=0.164)$ or gender $(\mathrm{CMH}, P=0.565)$. Categorizing the subjects into three age groups

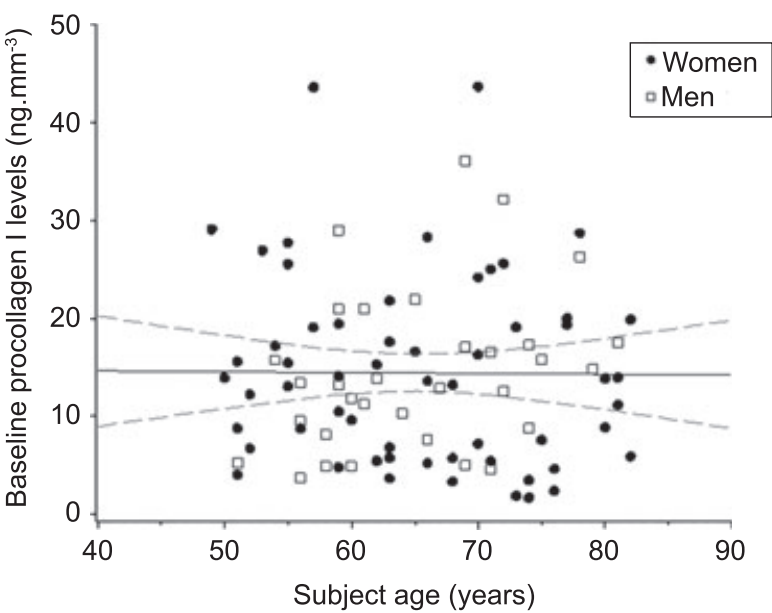

Figure 2 Distribution of baseline procollagen I levels in human forearm skin as a function of age and gender. Skin procollagen I levels of 91 individuals were measured by ELISA and plotted as a function of age and gender. Detailed characteristics of the cohort are summarized in Table 2. Black solid line represents regression line and dotted lines, the $95 \%$ confidence interval.

( $\leq 60$ years, $60-70$ years, $\geq 70$ years) showed no significant differences in improvement between the respective age groups $(\mathrm{CMH}, P=0.427)$. However, a significant difference in improvement was found among groups of subjects with hypo-collagenesis compared to normo-collagenesis ( $\mathrm{CMH}, \quad P=0.0012$; Fig. 3a). Specifically, we found that $65 \%$ (26 of 40 ) of the subjects with hypo-collagenesis showed improvement upon treatment, compared to improvement in only $22 \%$ (25 of 32) of normo-collagenesis subjects (Table 3). CMH-adjusted odds ratios indicated that, controlling for treatment among the five studies, subjects with hypo-collagenesis improved 6.4 times more often than subjects with normo-collagenesis (odds ratio confidence interval 1.93, 21.12). As shown in Figure $3 b$, we also found a highly significant, negative correlation between collagenesis levels and degree of improvement after treatment (Pearson's correlation coefficient $-0.43, \quad n=91, \quad P<0.0001)$. Taken together, these results demonstrate that subjects with hypo-collagenesis are more likely to have a positive response to anti-aging cosmeceuticals than subjects who have normo-collagenesis levels in their skin.

\section{Discussion}

We found that topical vitamin $\mathrm{C}$ was associated with a significant increase in collagen production in subjects with hypo-collagenesis, as measured by below-average steady-state procollagen I levels. In contrast, topical 
Table 3 Treatment outcome of increased procollagen level depends on baseline procollagen level

\begin{tabular}{|c|c|c|c|c|}
\hline \multirow[b]{2}{*}{$\begin{array}{l}\text { Variable } \\
\text { tested }\end{array}$} & \multicolumn{2}{|c|}{ Subject distribution* } & \multirow{2}{*}{$\begin{array}{l}\text { Improvement } \\
\text { associated } \\
\text { with variable? }\end{array}$} & \multirow[b]{2}{*}{$P$-value } \\
\hline & $\begin{array}{l}\text { No } \\
\text { improvement }\end{array}$ & Improvement & & \\
\hline \multicolumn{5}{|l|}{ Gender } \\
\hline Males & $17(19 \%)$ & $16(18 \%)$ & No & 0.565 \\
\hline Females & $35(38 \%)$ & $23(25 \%)$ & & \\
\hline \multicolumn{5}{|l|}{ Age } \\
\hline $\begin{array}{l}\text { Range } \\
\text { (years) }\end{array}$ & $49-82$ & $50-82$ & No & $0.164^{\dagger}$ \\
\hline \multicolumn{5}{|c|}{ Baseline procollagen } \\
\hline Low & $22(24 \%)$ & $31(34 \%)$ & Yes & 0.001 \\
\hline High & $30(33 \%)$ & $8(9 \%)$ & & \\
\hline
\end{tabular}

*Number of subjects ( $\%$ of total).

†Results of Pearson's correlation.

vitamin C had no effects on procollagen production in individuals with normo-collagenesis. Retrospective meta-analysis of similar studies with four additional over-the-counter cosmeceuticals yielded similar findings, that is, hypo-collagenesis significantly influenced treatment outcome regardless of age, gender, or cosmeceutical. Hypo-collagenesis therefore emerges as the unique parameter capable of use in cosmetology as a basis for product testing and developing custom formula skin care.

Our subject population was aged 49 to 82 , representative of typical cosmeceutical consumers. There are a couple of commonly held assumptions about the impact of cosmeceuticals on skin appearance. The first assumption is that low baseline procollagen I levels are associated with the biggest improvement in procollagen production, yet this has previously never been shown. We have been able to demonstrate that the over-thecounter cosmeceuticals we tested most benefited those with relatively severe photodamage, not those with minimal photodamage. The second assumption is that cosmeceuticals will have greater efficacy in older subjects, whether the visible markers of their aging is due to photoaging or to intrinsic aging. Our results prove this to be false. These cosmeceuticals are beneficial to the subset of individuals who have biochemical damage evidenced by hypo-collagenesis, not necessarily the subset who is oldest.

Further studies will be required to precisely define clinical features that are best associated with hypocollagenesis in photoaged skin. Measurement of skin procollagen I levels prior to treatment demonstrated a strong interindividual variation among our cohort. Reductions in both type I and type III procollagens in

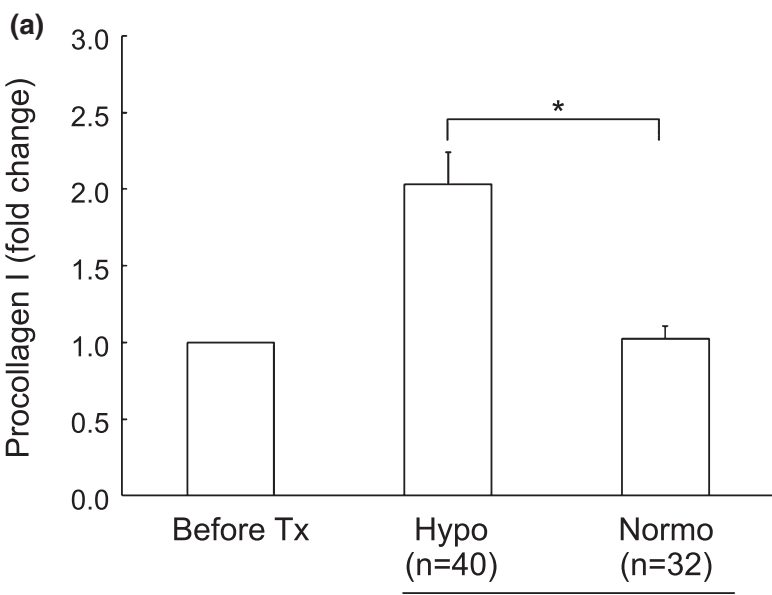

(b)

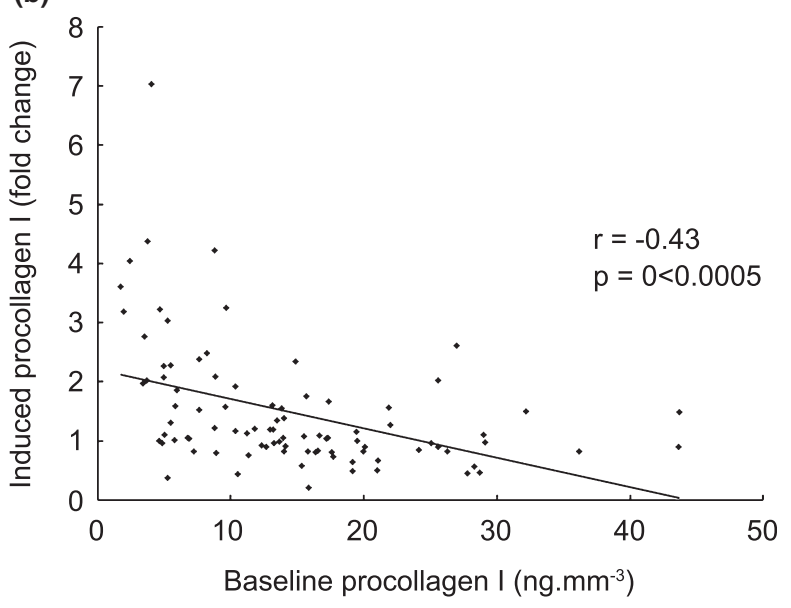

Figure 3 Cosmeceutical treatment outcome is different for hypoand normo-collagenesis subjects (a) Procollagen I levels in skin biopsies taken before and after treatment (Tx) with one of five cosmeceutical preparations on one randomly chosen photoaged forearm for 2 weeks. Subjects were retrospectively divided in groups based on their initial collagenesis levels (hypo- or normocollagenesis). *: $\mathrm{P}<0.01$ between the two groups, controlling for the different treatments (b) Pearson's correlation suggests an inverse relationship between collagenesis levels and improvement with cosmeceutical (correlation coefficient $=-0.43, \mathrm{n}=91$, $\mathrm{P}<0.0001)$.

skin have been shown to occur in severely photodamaged skin when assessed by immunohistochemistry or Western blot analysis. ${ }^{9}$ These measurement techniques established that skin with milder photodamage has normo-collagenesis levels, similar to levels found in sun-protected skin. ${ }^{9}$ Our procollagen I measurements, performed with a more sensitive technique (ELISA), confirmed strong interindividual variation in the subject group. Recent review of the literature indicates that no single clinical assessment can accurately quantify 
the degree of photodamage in skin. ${ }^{14}$ Although photonumeric scales exist for facial and forearm photoaging, ${ }^{15,16}$ hypo-collagenesis has never been correlated with any clinical feature. It will be interesting to collect precise clinical data such as degree/amount of rhytides, sallowness, mottled pigmentation, lentigines, and telangiectasia to establish which clinical feature is best associated with hypo-collagenesis. A non-invasive tool as described by Hermanns-Lê and colleagues to further investigate hypo-collagenesis would be desirable in future investigations of cosmeceuticals. ${ }^{17}$

It is conceivable that a treatment time longer than 2 weeks would have allowed for increased procollagen I production in the skin of subjects with normo-collagenesis. Additional studies will be necessary to formally test this possibility. However, shortening the treatment period limited the possibility of alterations in collagen production that were not directly produced by the cosmeceutical treatment. Furthermore, for topical vitamin C, induction in procollagen I achieved after 2-week treatment in our study was similar to that obtained after 6 months of daily application. ${ }^{12}$

Comparison between cosmeceuticals was not appropriate due to study design. Such a comparison would require subject randomization, determining baseline procollagen levels prior to study entry, and randomizing subjects with hypo- and normo-collagenesis to receive one of the five cosmeceuticals. Such randomization was not performed in the studies presented here. However, potential bias was limited by conducting all studies in the same location, by the same investigators, using the same reagents, and under similar conditions. Comparison with respective vehicle was also not possible due to the proprietary nature of the vehicles used in commercial products; regardless, statistical analysis shows that the nature of the topical agent did not influence treatment outcome. Also, it is possible that treatment outcome might be due to the action of cream application itself, as opposed to active ingredient of the cream. Further rigorous double-blinded placebocontrolled studies will be required to answer these questions.

This study associates a known photoaging biomarker to anti-aging cosmeceutical response. Our results demonstrate that hypo-collagenesis is a strong predictor of anti-aging cosmeceutical response as measured by increased procollagen I levels, and that age, gender, and type of cosmeceutical (among those tested) are not. This and potential additional validated indicators are likely to be useful in the design and testing of custom formula skin preparations in the future.

\section{Acknowledgments}

The authors wish to thank Suzan Rehbine, LPN, for help with subject recruitment and tissue procurement. We would also like to thank Laura Klem (Center for Statistical Consultation and Research, University of Michigan) for helpful discussion, Stephanie Cooke and Irina Bondarenko for technical help, and Jackie Giletto for help with manuscript preparation.

\section{Conflict of interest}

No authors reported any financial conflict of interest.

\section{References}

1 Kligman AM. Early destructive effect of sunlight on human skin. JAMA 1969; 210: 2377-80.

2 Fisher GJ, Kang S, Varani J et al. Mechanisms of photoaging and chronological skin aging. Arch Dermatol 2002; 138: $1462-70$.

3 Fisher GJ, Wang ZQ, Datta SC et al. Pathophysiology of premature skin aging induced by ultraviolet light. $N$ Engl J Med 1997; 337: 1419-28.

4 Fisher GJ, Voorhees JJ. Molecular mechanisms of photoaging and its prevention by retinoic acid: ultraviolet irradiation induces MAP kinase signal transduction cascades that induce Ap-1-regulated matrix metalloproteinases that degrade human skin in vivo. J Investig Dermatol Symp Proc 1998; 3: 61-8.

5 Lambert CA, Soudant EP, Nusgens BV, Lapiere CM. Pretranslational regulation of extracellular matrix macromolecules and collagenase expression in fibroblasts by mechanical forces. Lab Invest 1992; 66: 444-51.

6 Rittie L, Fisher G, Voorhees JJ. Aging and Photoaging of the Skin. In: Krieg T, Bickers DR, Miyachi Y eds. Therapy of Skin Diseases. Berlin-Heidelberg-New York: SpringerVerlag; 2010: pp. 705-16.

7 Draelos Z. The future of cosmeceuticals: an interview with Albert Kligman, MD, PhD. Dermatol Surg 2005; 31: 890-1.

8 Rittie L, Fisher G, Voorhees JJ. Retinoid therapy for photoaging. In: BA Gilchrest, J Krutman, eds. Skin Ageing. Berlin-Heidelberg-New York: Springer-Verlag; 2006: pp. 143-56.

9 Talwar HS, Griffiths CE, Fisher GJ et al. Reduced type I and type III procollagens in photodamaged adult human skin. J Invest Dermatol 1995; 105: 285-90.

10 Rittié L, Kang S, Voorhees JJ, Fisher GJ. Induction of collagen by estradiol: difference between sun-protected and photodamaged human skin in vivo. Arch Dermatol 2008; 144: 1129-40.

11 Orringer JS, Rittie L, Hamilton T et al. Intraepidermal erbium:YAG laser resurfacing: impact on the dermal matrix. J Am Acad Dermatol 2011; 64: 119-28. 
12 Nusgens BV, Humbert P, Rougier A et al. Topically applied vitamin $\mathrm{C}$ enhances the mRNA level of collagens I and III, their processing enzymes and tissue inhibitor of matrix metalloproteinase 1 in the human dermis. J Invest Dermatol 2001; 116: 853-9.

13 Humbert PG, Haftek M, Creidi P et al. Topical ascorbic acid on photoaged skin. Clinical, topographical and ultrastructural evaluation: double-blind study vs. placebo. Exp Dermatol 2003; 12: 237-44.

14 Baillie L, Askew D, Douglas N, Soyer HP. Strategies for assessing the degree of photodamage to skin: a systematic review of the literature. Br J Dermatol 2011; 165: 735-42.
15 Griffiths CE, Wang TS, Hamilton TA et al. A photonumeric scale for the assessment of cutaneous photodamage. Arch Dermatol 1992; 128: 347-51.

16 McKenzie NE, Saboda K, Duckett LD et al. Development of a photographic scale for consistency and guidance in dermatologic assessment of forearm sun damage. Arch Dermatol 2011; 147: 31-6.

17 Hermanns-Le T, Jonlet F, Scheen A, Pierard GE. Ageand body mass index-related changes in cutaneous shear wave velocity. Exp Gerontol 2001; 36: 363-72. 